\title{
ON THE DISTRIBUTION OF STORAGE PROCESSES FROM THE CLASS $V(\varphi, \psi)$
}

UDC 519.21

\author{
R. E. YAMNENKO AND O. S. SHRAMKO
}

Abstract. Estimates for the distribution of a storage process

$$
Q(t)=\sup _{s \leq t}(X(t)-X(s)-(f(t)-f(s)))
$$

are obtained in the paper, where $(X(t), t \in T)$ is a stochastic process belonging to the class $V(\varphi, \psi)$ and where the service output rate $f(t)$ is a continuous function. In particular, the results hold if $(X(t), t \in T)$ is a Gaussian process. Several examples of applications of the results obtained in the paper are given for sub-Gaussian stationary stochastic processes.

\section{INTRODUCTION}

A considerable number of papers are devoted nowadays to studies of the behavior of the data traffic in computer networks (see, for example, [1, 2, 4, 8, 10]). A special place in this topic is occupied by the problem of estimating the reliability when transferring the data. Several stochastic models of the data traffic have been studied over recent years. Among those models are stochastic processes belonging to some classes describing a specific traffic of the data, namely Markov stochastic processes, processes with long as well as with short memory, automodel processes, Gaussian processes, especially fractional Brownian motion, autoregressive integrated moving average processes (ARIMA), etc.

The current paper deals with the distribution of a storage process generated by a stochastic process $\{X(t), t \in T\}$ belonging to a rather general class $V(\varphi, \psi)$ containing, for example, Gaussian stochastic processes.

Let $T$ be a set of parameters.

The process under consideration is defined as follows.

Definition 1.1. A process $Q(t)=\{Q(t), t \in T\}$ given by

$$
Q(t)=\sup _{s \leq t}(X(t)-X(s)-(f(t)-f(s))), \quad s, t \in T
$$

is called a storage process generated by the input $X(t)=\{X(t), t \in T\}$ and a service output rate $f(t)$.

Assume that a work arrives to a server. If more work arrives than can be processed, the surplus waits in the queue buffer of length $x \geq 0$. A work that arrives after the buffer overflows is lost. An important problem of queuing theory is to estimate the overflow probability, namely

$$
\mathrm{P}\{Q(t)>x\} .
$$

2010 Mathematics Subject Classification. Primary 60G07; Secondary 60K25.

Key words and phrases. Metric entropy, queue, storage process, estimate of a distribution, subGaussian process. 
The same problem is stated in terms of the ruin probability for the corresponding risk process in risk theory.

We consider the case where the input process $X(t)$ belongs to the class $V(\varphi, \psi)$. Recall that $V(\varphi, \psi)$ contains the class of $\varphi$-sub-Gaussian stochastic processes. More detail and results concerning the processes of $V(\varphi, \psi)$ can be found in [3, 13, and other sources are included in the list of references at the end of the paper.

The paper is organized as follows. Section 2 contains necessary definitions and results concerning $\varphi$-sub-Gaussian random variables and stochastic processes. Section 3 is devoted to the main results proved with the help of the metric entropy method. An application of the results obtained in Section 2 to stationary sub-Gaussian stochastic processes is given in Section 3 .

\section{NeCESSARY DEFINITIONS AND RESUlts}

Let $\{\Omega, \mathcal{B}, P\}$ be a standard probability space and let $T$ be a space of parameters.

\subsection{Orlicz $N$-functions, Young-Fenchel transform.}

Definition 2.1 ([3]). A function $U=\{U(x), x \in \mathbb{R}\}$ is called an Orlicz $N$-function if $U$ is continuous, even, and such that

1) $U(0)=0$

2) $U(x)$ increases for $x>0$,

3) $U(x) / x \rightarrow 0$ as $x \rightarrow 0$ and $U(x) / x \rightarrow \infty$ as $x \rightarrow \infty$.

Condition Q. We say that condition $\mathbf{Q}$ holds for an Orlicz $N$-function $\varphi$ if

$$
\liminf _{x \rightarrow 0} \frac{\varphi(x)}{x^{2}}=c>0 .
$$

Definition 2.2. We say that an $N$-function $\varphi_{1}$ is subordinated to an $N$-function $\varphi_{2}$ and denote $\varphi_{1} \prec \varphi_{2}$ if there are some constants $c>0$ and $x_{0}>0$ such that $\varphi_{1}(x)<\varphi_{2}(c x)$ for $x>x_{0}$.

We say that two $N$-functions $\varphi_{1}$ and $\varphi_{2}$ are equivalent if $\varphi_{1} \prec \varphi_{2}$ and $\varphi_{2} \prec \varphi_{1}$.

Definition 2.3. Let $\varphi=\{\varphi(x), x \in \mathbb{R}\}$ be an $N$-function. Then

$$
\varphi^{*}(x)=\sup _{y \in \mathbb{R}}(x y-\varphi(y))
$$

is called the Young-Fenchel transform of the function $\varphi$.

\section{2. $\varphi$-sub-Gaussian random variables and stochastic processes.}

Definition $2.4([3])$. Let $\varphi$ be an $N$-function satisfying condition $\mathbf{Q}$. We say that a

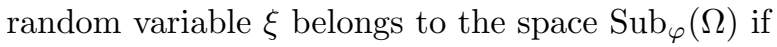

1) $\mathrm{E} \xi=0$,

2) $\mathrm{E} \exp \{\lambda \xi\}$ is finite for all $\lambda \in \mathbb{R}$, and

3) there exists a constant $a>0$ such that

$$
\mathrm{E} \exp \{\lambda \xi\} \leq \exp \{\varphi(\lambda a)\}
$$

for all $\lambda \in \mathbb{R}$.

Theorem 2.1 ([3]). The space $\operatorname{Sub}_{\varphi}(\Omega)$ is a Banach space with respect to the norm

$$
\tau_{\varphi}(\xi)=\sup _{\lambda>0} \frac{\varphi^{(-1)}(\log \mathrm{E} \exp \{\lambda \xi\})}{\lambda},
$$


where $\varphi^{(-1)}$ is the inverse function to $\varphi$. In addition,

$$
\mathrm{E} \exp \{\lambda \xi\} \leq \exp \left\{\varphi\left(\lambda \tau_{\varphi}(\xi)\right)\right\}
$$

for all $\lambda \in \mathbb{R}$.

Lemma 2.1 ([13]). Let a random variable $\xi \in \operatorname{Sub}_{\varphi}(\Omega)$ be such that $\tau_{\varphi}(\xi)>0$ and let $\varepsilon>0$. Then

$$
\begin{gathered}
\mathrm{P}\{\xi>\varepsilon\} \leq \exp \left\{-\varphi^{*}\left(\frac{\varepsilon}{\tau_{\varphi}(\xi)}\right)\right\}, \\
\mathrm{P}\{\xi<-\varepsilon\} \leq \exp \left\{-\varphi^{*}\left(\frac{\varepsilon}{\tau_{\varphi}(\xi)}\right)\right\}, \\
\mathrm{P}\{|\xi|>\varepsilon\} \leq 2 \exp \left\{-\varphi^{*}\left(\frac{\varepsilon}{\tau_{\varphi}(\xi)}\right)\right\} .
\end{gathered}
$$

Lemma $2.2([13])$. Let a random variable $\xi$ belong to the space $\operatorname{Sub}_{\varphi}(\Omega)$. Then

$$
\left|\mathrm{E} \xi^{k}\right| \leq \mathrm{E}|\xi|^{k} \leq 2\left(\tau_{\varphi}(\xi)\right)^{k} \frac{e^{k}}{\left(\varphi^{(-1)}(k)\right)^{k}} k !
$$

for $k=1,2, \ldots$.

Example $2.1([3])$. Let $\xi$ be a centered Gaussian random variable, that is, $\xi \in N\left(0, \sigma^{2}\right)$. Then $\xi$ belongs to the space $\operatorname{Sub}_{x^{2} / 2}(\Omega)$ and $\tau(\xi)=\left(E \xi^{2}\right)^{1 / 2}$.

Definition 2.5. A stochastic process $X=\{X(t), t \in T\}$ is called $\varphi$-sub-Gaussian if the random variables $X(t), t \in T$, are $\varphi$-sub-Gaussian. We write $X(t) \in \operatorname{Sub}_{\varphi}(\Omega)$ in this case.

If $\varphi(x)=x^{2} / 2$, then the processes $X(t) \in \operatorname{Sub}_{\varphi}(\Omega)$ are called sub-Gaussian.

Example 2.2. Every centered Gaussian stochastic process is a sub-Gaussian process.

\subsection{Strictly $\varphi$-sub-Gaussian random variables and stochastic processes.}

Definition 2.6 ([6]). A family $\Delta$ of random variables belonging to the space $\operatorname{Sub}_{\varphi}(\Omega)$ is called strictly $\varphi$-sub-Gaussian if there exists a constant $C_{\Delta}>0$ such that

$$
\tau_{\varphi}\left(\sum_{i \in I} \lambda_{i} \xi_{i}\right) \leq C_{\Delta}\left(\mathrm{E}\left(\sum_{i \in I} \lambda_{i} \xi_{i}\right)^{2}\right)^{1 / 2}
$$

for all finite sets $I$, all $\xi_{i} \in \Delta, i \in I$, and all $\lambda_{i} \in \mathbb{R}^{1}$.

The constant $C_{\Delta}$ is called a defining constant for the family $\Delta$.

Theorem $2.2([6])$. Let $\Delta$ be a strictly $\varphi$-sub-Gaussian family. Then the linear closure of the family $\Delta$ in the space $\operatorname{Sub}_{\varphi}(\Omega)$ (or in $L_{2}(\Omega)$ ) also is a strictly $\varphi$-sub-Gaussian family with the same defining constant.

The linear closure of strictly $\operatorname{Sub}_{\varphi}(\Omega)$ random variables forms the space of strictly $\operatorname{Sub}_{\varphi}(\Omega)$ random variables. This space is denoted by $\operatorname{SSub}_{\varphi}(\Omega)$.

Definition 2.7. A stochastic process $X=\{X(t), t \in T\}$ is called strictly $\varphi$-sub-Gaussian if the random variables $\{X(t), t \in T\}$ form a strictly $\varphi$-sub-Gaussian family.

The defining constant of this family is called the defining constant of the process $X$ and is denoted by $C_{X}$. 


\subsection{Stochastic processes belonging to the class $V(\varphi, \psi)$.}

Definition 2.8 ([13]). Let $\varphi \prec \psi$ be two Orlicz $N$-functions. We say that a stochastic process $X=\{X(t), t \in T\}$ belongs to the class $V(\varphi, \psi)$ if the random variable $X(t)$

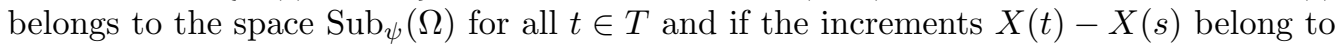

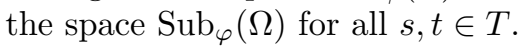

Example 2.3 ([3]). Every sub-Gaussian stochastic process belongs to the class $V(\varphi, \varphi)$, where $\varphi=x^{2} / 2$.

Example $2.4([13])$. Let

$$
X(t)=\xi_{0}+\sum_{k=1}^{\infty} \xi_{k} f_{k}(t),
$$

where the random variable $\xi_{0}$ belongs to $\operatorname{Sub}_{\psi}(\Omega),\left\{\xi_{k}, k=1,2, \ldots\right\} \in \operatorname{Sub}_{\varphi}(\Omega)$, and where $\varphi$ is an Orlicz $N$-function such that $\varphi(\sqrt{x})$ is even and

$$
\sum_{k=1}^{\infty} \tau_{\varphi}\left(\xi_{k}\right)\left|f_{k}(t)\right|<\infty .
$$

Then the stochastic process $X(t)$ belongs to the class $V(\varphi, \psi)$.

\section{MAin RESUlts}

Let $(T, \rho)$ be a pseudo-metric (metric) separable space equipped with a pseudo-metric (metric) $\rho$. Recall that a pseudo-metric satisfies all the conditions for a metric except the following one: if $\rho(t, s)=0$, then $t=s$. The latter means that the set $\{(t, s): \rho(t, s)=0\}$ is possibly wider than the "diagonal" $\{(t, s): t=s\}$ (see [3]).

Consider a separable stochastic process $X=\{X(t), t \in T\}$ belonging to the class $V(\varphi, \psi)$. Assume that there exists a continuous increasing function $\sigma=\{\sigma(h), h>0\}$ such that $\sigma(h) \rightarrow 0$ as $h \rightarrow 0$ and

$$
\sup _{\rho(t, s) \leq h} \tau_{\varphi}(X(t)-X(s)) \leq \sigma(h) .
$$

Note that the function

$$
\sigma(h)=\sup _{\rho(t, s) \leq h} \tau_{\varphi}(X(t)-X(s))
$$

can be used on the right hand side of (7) if the process $X(t)$ is continuous in the norm $\tau_{\varphi}(\cdot)$.

Let $B$ be a compact set, $B \subset T$. In what follows we use the following notation:

1) $\gamma(u)=\tau_{\psi}(X(u))$;

2) $B_{t}=\{u \in B: u \leq t\}$;

3) $\beta>0$ is a number such that $\beta \leq \sigma\left(\inf _{s \in B} \sup _{t \in B} \rho(t, s)\right)$;

4) $\zeta_{\varphi}(v)=\frac{v}{\varphi^{(-1)}(v)}$;

5) $N_{B}(u)=N_{(B, \rho)}(u)$ is the metric capacity of the space $(B, \rho)$, that is, the minimum number of closed balls of radius $u$ covering the space $(B, \rho)$;

6) $H_{B}(u)=H_{(B, \rho)}(u)=\ln N_{B}(u)$ is the metric entropy of the space $(B, \rho)$.

Let a separable stochastic process $X=\{X(t), t \in B\}$ belong to the class $V(\varphi, \psi)$ and be defined on a compact set $B$. Assume that $\gamma(u)<\infty$ and that $f=\{f(t), t \in B\}$ is a continuous function. The following result contains conditions for the boundedness of the stochastic process

$$
Q(t)=\sup _{s \leq t}(X(t)-X(s)-(f(t)-f(s))), \quad s, t \in B .
$$

Lemma 3.1 also provides the bounds for the exponential moments of this process. 
Lemma 3.1. Let $f=\{f(t), t \in B\}$ be a continuous function such that

$$
|f(u)-f(w)| \leq \delta(\rho(u, w)),
$$

where $\delta=\{\delta(s), s>0\}$ is a nonnegative increasing function. Let $\left\{q_{k}, k=1,2, \ldots\right\}$ be a sequence such that $q_{k}>1, k \geq 1$, and

$$
\sum_{k=1}^{\infty} \frac{1}{q_{k}} \leq 1 .
$$

Then

$$
\begin{aligned}
\mathrm{E} \exp \{\lambda Q(t)\} \leq & W(\lambda, t, p)\left(\prod_{k=2}^{\infty}\left(N_{B_{t}}\left(\sigma^{(-1)}\left(\beta p^{k}\right)\right)\right)^{1 / q_{k}}\right) \\
& \times\left(\prod_{k=2}^{\infty} \exp \left\{\frac{1}{q_{k}} \varphi\left(\lambda q_{k} \beta p^{k-1}\right)+\lambda \delta\left(\sigma^{(-1)}\left(\beta p^{k-1}\right)\right)\right\}\right)
\end{aligned}
$$

for all $\lambda>0$ and $p \in(0,1)$, where

$$
W(\lambda, t, p)=\min \left\{X_{\lambda, t, p} ; Y_{\lambda, t, p}\right\}
$$

and

$$
\begin{aligned}
& X_{\lambda, t, p}=\left(\sum_{j=1}^{N_{B_{t}}\left(\sigma^{(-1)}(\beta p)\right)} \exp \left\{\varphi\left(q_{1} \lambda \sigma\left(2 j \sigma^{(-1)}(\beta p)\right)\right)+q_{1} \lambda \delta\left(2 j \sigma^{(-1)}(\beta p)\right)\right\}\right)^{1 / q_{1}}, \\
& Y_{\lambda, t, p}=\left(N_{B_{t}}\left(\sigma^{(-1)}(\beta p)\right)\right)^{\frac{1}{q_{1}}} \\
& \times \inf _{w>1} \exp \left\{\frac{\psi\left(w q_{1} \lambda \gamma(t)\right)}{w q_{1}}\right. \\
&\left.\quad+\max _{u \in B_{t}}\left\{\frac{(w-1) \psi\left(\frac{w}{w-1} q_{1} \lambda \gamma(u)\right)}{w q_{1}}+\lambda f(u)\right\}-\lambda f(t)\right\} .
\end{aligned}
$$

Remark 3.1. Clearly, inequality (9) makes sense if the denominators on the right hand side are finite.

Proof. Denote by $V_{\varepsilon_{k}}$ the set of centers of closed balls of radius

$$
\varepsilon_{k}=\sigma^{(-1)}\left(\beta p^{k}\right), \quad p \in(0,1), \quad k=0,1,2, \ldots,
$$

that form the minimal covering of the space $(B, \rho)$. The number of sets in the set $V_{\varepsilon_{k}}$ is equal to $N_{B}\left(\varepsilon_{k}\right)$.

Note that $X(t)$ and thus $X_{f}(t)=X(t)-f(t)$ are separable processes.

Lemma 2.1 and assumption (7) imply that

$$
\begin{aligned}
\mathrm{P}\{|X(t)-X(s)|>\varepsilon\} & \leq 2 \exp \left\{-\varphi^{*}\left(\frac{\varepsilon}{\tau_{\varphi}(X(t)-X(s))}\right)\right\} \\
& \leq 2 \exp \left\{-\varphi^{*}\left(\frac{\varepsilon}{\sigma(\rho(t, s))}\right)\right\}
\end{aligned}
$$

for all $\varepsilon>0$. Hence the process $X$ and thus $X_{f}$, too, are continuous in probability. If a separable stochastic process defined on $(B, \rho)$ is continuous in probability, then every finite and everywhere dense set with respect to $\rho$ is the set of separability for this 
process. Thus $V=\bigcup_{k=1}^{\infty} V_{\varepsilon_{k}}$ is a set of $\rho$-separability of the process $X_{f}$. Moreover, with probability one,

$$
Q(t)=\sup _{s \leq t, s \in B}\left(X_{f}(t)-X_{f}(s)\right)=\sup _{s \leq t, s \in V}\left(X_{f}(t)-X_{f}(s)\right) .
$$

Consider the mapping $\alpha_{n}=\left\{\alpha_{n}(s), n=0,1, \ldots\right\}$ of the set $V$ to $V_{\varepsilon_{n}}$ such that $\alpha_{n}(s)$ is a point of the set $V_{\varepsilon_{n}}$ for which $\rho\left(s, \alpha_{n}(s)\right)<\varepsilon_{n}$. If $s \in V_{\varepsilon_{n}}$, then $\alpha_{n}(s)=s$. If there are several points in the set $V_{\varepsilon_{n}}$ such that $\rho\left(s, \alpha_{n}(s)\right)<\varepsilon_{n}$, then we choose an arbitrary point among them and denote it by $\alpha_{n}(s)$.

Chebyshev's inequality, Lemma 2.1, and assumption (7) imply that

$$
\begin{aligned}
\mathrm{P}\left\{\left|X(s)-X\left(\alpha_{n}(s)\right)\right|>p^{n / 2}\right\} & \leq \frac{\mathrm{E}\left(X(s)-X\left(\alpha_{n}(s)\right)\right)^{2}}{p^{n}} \\
& \leq \frac{c^{2} \tau_{\varphi}^{2}\left(X(s)-X\left(\alpha_{n}(s)\right)\right)}{p^{n}} \leq \frac{c^{2} \sigma^{2}\left(\varepsilon_{n}\right)}{p^{n}}=c^{2} \beta^{2} p^{n}
\end{aligned}
$$

where

$$
c=\frac{2 e}{\varphi^{(-1)}(2)} .
$$

The latter inequality means that

$$
\sum_{n=1}^{\infty} \mathrm{P}\left\{\left|X(s)-X\left(\alpha_{n}(s)\right)\right|>p^{n / 2}\right\}<\infty
$$

Now the Borel-Cantelli lemma implies that $X(s)-X\left(\alpha_{n}(s)\right) \rightarrow 0$ as $n \rightarrow \infty$ with probability one. Since the function $f$ is continuous,

$$
X_{f}(s)-X_{f}\left(\alpha_{n}(s)\right) \rightarrow 0 \quad \text { as } n \rightarrow \infty
$$

with probability one. The set $V$ is countable; hence $X(s)-X\left(\alpha_{n}(s)\right) \rightarrow 0$ with probability one as $n \rightarrow \infty$ uniformly in $s$.

Let $s$ be an arbitrary point of the set $V$. Let, for all $m \geq 1$,

$$
s_{m}=\alpha_{m}(s), \quad s_{m-1}=\alpha_{m-1}\left(s_{m}\right), \quad \ldots, \quad s_{1}=\alpha_{1}\left(s_{2}\right) .
$$

Then

$$
X_{f}(s)=X_{f}\left(s_{1}\right)+\sum_{k=2}^{m}\left(X_{f}\left(s_{k}\right)-X_{f}\left(s_{k-1}\right)\right)+X_{f}(s)-X_{f}\left(\alpha_{m}(s)\right)
$$

for all $m \geq 2$ and similarly

(13) $-X_{f}(s) \leq-\min _{u \in V_{\varepsilon_{1}}} X_{f}(u)-\sum_{k=2}^{m} \min _{u \in V_{\varepsilon_{k}}}\left(X_{f}(u)-X_{f}\left(\alpha_{k-1}(u)\right)\right)-X_{f}(s)+X_{f}\left(\alpha_{m}(s)\right)$.

Relations (8) and (13) imply that

$$
\begin{aligned}
Q(t) & =\sup _{\substack{s \leq t, s \in V}}\left(X_{f}(t)-X_{f}(s)\right) \\
& \leq \lim _{m \rightarrow \infty} \inf \left(X_{f}(t)-\min _{\substack{u \leq t, u \in V_{\varepsilon_{1}}}} X_{f}(u)-\sum_{k=2}^{m} \min _{\substack{u \leq t, u \in V_{\varepsilon_{k}}}}\left(X_{f}(u)-X_{f}\left(\alpha_{k-1}(u)\right)\right)\right. \\
& \left.-X_{f}(s)+X_{f}\left(\alpha_{m}(s)\right)\right)
\end{aligned}
$$

with probability one. 
Now Hölder's inequality, (12), (14), and Fatou's lemma yield

(15)

$$
\begin{aligned}
& \mathrm{E} \exp \{\lambda Q(t)\} \\
& \leq \mathrm{E} \lim _{m \rightarrow \infty} \inf \exp \left\{\lambda\left(X_{f}(t)-\min _{\substack{u \leq t, u \in V_{\varepsilon_{1}}}} X_{f}(u)-\sum_{k=2}^{m} \min _{\substack{u \leq t, u \in V_{\varepsilon_{k}}}}\left(X_{f}(u)-X_{f}\left(\alpha_{k-1}(u)\right)\right)\right)\right\} \\
& \leq \lim _{m \rightarrow \infty} \inf E \exp \left\{\lambda\left(X_{f}(t)-\min _{\substack{u \leq t, u \in V_{\varepsilon_{1}}}} X_{f}(u)-\sum_{k=2}^{m} \min _{\substack{u \leq t, u \in V_{\varepsilon_{k}}}}\left(X_{f}(u)-X_{f}\left(\alpha_{k-1}(u)\right)\right)\right)\right\} \\
& \leq \lim _{m \rightarrow \infty} \inf \left(\left(\operatorname{E} \exp \left\{q_{1} \lambda\left(X_{f}(t)-\min _{\substack{u \leq t, u \in V_{\varepsilon_{1}}}} X_{f}(u)\right)\right\}\right)^{1 / q_{1}}\right. \\
& \left.\times \prod_{k=2}^{m}\left(\mathrm{E} \exp \left\{q_{k} \lambda \max _{\substack{u \leq t, u \in V_{\varepsilon_{k}}}}\left(X_{f}\left(\alpha_{k-1}(u)\right)-X(u)\right)\right\}\right)^{1 / q_{k}}\right) \\
& \leq\left(\operatorname{E} \exp \left\{q_{1} \lambda\left(X_{f}(t)-\min _{\substack{u \leq t, u \in V_{\varepsilon_{1}}}} X_{f}(u)\right)\right\}\right)^{1 / q_{1}} \\
& \times \prod_{k=2}^{\infty}\left(\mathrm{E} \exp \left\{q_{k} \lambda \max _{\substack{u \leq t, u \in V_{\varepsilon_{k}}}}\left(X_{f}\left(\alpha_{k-1}(u)\right)-X(u)\right)\right\}\right)^{1 / q_{k}} \\
& =I_{1} \cdot \prod_{k=2}^{\infty} I_{k}
\end{aligned}
$$

for all $\lambda>0$.

Every term on the right hand side of (15) is considered separately. Theorem 2.1 and assumption (7) imply that

$$
\begin{aligned}
E \exp \left\{q_{1} \lambda(X(t)-X(u))\right\} & \leq \exp \left\{\varphi\left(q_{1} \lambda \tau_{\varphi}(X(t)-X(u))\right)\right\} \\
& \leq \exp \left\{\varphi\left(q_{1} \lambda \sigma(\rho(t, u))\right)\right\}
\end{aligned}
$$

Then the condition $|f(u)-f(w)| \leq \delta(\rho(u, w))$ yields

$$
\begin{aligned}
\left(I_{1}\right)^{q_{1}} \leq & \sum_{\substack{u \leq t, u \in V_{\varepsilon_{1}}}} \operatorname{Eexp}\left\{q_{1} \lambda(X(t)-X(u))\right\} \exp \left\{-q_{1} \lambda(f(t)-f(u))\right\} \\
\leq & \sum_{\substack{u \leq t, u \in V_{\varepsilon_{1}}}} \exp \left\{\varphi\left(q_{1} \lambda \sigma(\rho(t, u))\right)+q_{1} \lambda \delta(\rho(t, u))\right\} \\
& \leq \sum_{j=1}^{N_{B_{t}}\left(\varepsilon_{1}\right)} \exp \left\{\varphi\left(q_{1} \lambda \sigma\left(2 j \varepsilon_{1}\right)\right)+q_{1} \lambda \delta\left(2 j \varepsilon_{1}\right)\right\} .
\end{aligned}
$$


On the other hand, the Hölder inequality and (4) imply that, for all $w>1$, (17)

$$
\begin{aligned}
\left(I_{1}\right)^{q_{1} \leq} & \sum_{\substack{u \leq t, u \in V_{\varepsilon_{1}}}}\left(\operatorname{E} \exp \left\{w q_{1} \lambda X(t)\right\}\right)^{\frac{1}{w}}\left(\mathrm{E} \exp \left\{-\frac{w}{w-1} q_{1} \lambda X(u)\right\}\right)^{1-\frac{1}{w}} \\
& \times \exp \left\{-q_{1} \lambda(f(t)-f(u))\right\} \\
\leq & \sum_{\substack{u \leq t, u \in V_{\varepsilon_{1}}}} \exp \left\{\frac{\psi\left(w q_{1} \lambda \gamma(t)\right)}{w}-q_{1} \lambda f(t)+\frac{(w-1) \psi\left(\frac{w}{w-1} q_{1} \lambda \gamma(u)\right)}{w}+q_{1} \lambda f(u)\right\} \\
\leq & N_{B_{t}}\left(\varepsilon_{1}\right) \exp \left\{\frac{\psi\left(w q_{1} \lambda \gamma(t)\right)}{w}-q_{1} \lambda f(t)\right\} \\
& \times \exp \left\{\max _{u \in B_{t}}\left(\frac{(w-1) \psi\left(\frac{w q_{1} \lambda \gamma(u)}{w-1}\right)}{w}+q_{1} \lambda f(u)\right)\right\} .
\end{aligned}
$$

Further, Theorem 2.1 and (7) imply that

$$
\mathrm{E} \exp \left\{q_{k} \lambda\left(X(u)-X\left(\alpha_{k-1}(u)\right)\right)\right\} \leq \exp \left\{\varphi\left(q_{k} \lambda \sigma\left(\varepsilon_{k-1}\right)\right)\right\} .
$$

Then

$$
\begin{aligned}
\left(I_{k}\right)^{q_{k} \leq} \leq & N_{B_{t}}\left(\varepsilon_{k}\right) \max _{\substack{u \leq t, u \in V_{\varepsilon_{k}}}} \operatorname{Eexp}\left\{q_{k} \lambda\left(X_{f}\left(\alpha_{k-1}(u)\right)-X(u)\right)\right\} \\
& \times \exp \left\{q_{k} \lambda\left[f(u)-f\left(\alpha_{k-1}(u)\right)\right]\right\} \\
\leq & N_{B_{t}}\left(\varepsilon_{k}\right) \max _{\substack{u \leq t, u \in V_{\varepsilon_{k}}}} \exp \left\{\varphi\left(q_{k} \lambda \sigma\left(\varepsilon_{k-1}\right)\right)+q_{k} \lambda \delta\left(\rho\left(u, \alpha_{k-1}(u)\right)\right)\right\} \\
\leq & N_{B_{t}}\left(\varepsilon_{k}\right) \exp \left\{\varphi\left(q_{k} \lambda \beta p^{k-1}\right)+q_{k} \lambda \delta\left(\sigma^{(-1)}\left(\beta p^{k-1}\right)\right)\right\} .
\end{aligned}
$$

Inequalities (15)- (18) complete the proof of Lemma 3.1

Theorem 3.1. Let all the assumptions of Lemma 3.1 hold. Then

$$
\mathrm{P}\{Q(t)>x\} \leq Z(p, t, x)
$$

for all $p \in(0,1)$ and $x>0$, where

$$
\begin{aligned}
Z(p, t, x)= & \inf _{\lambda>0} W(\lambda, t, p)\left(\prod_{k=2}^{\infty}\left(N_{B_{t}}\left(\sigma^{(-1)}\left(\beta p^{k}\right)\right)\right)^{\frac{1}{q_{k}}}\right) \\
& \times \exp \left\{\sum_{k=2}^{\infty}\left(\frac{1}{q_{k}} \varphi\left(\lambda q_{k} \beta p^{k-1}\right)+\lambda \delta\left(\sigma^{(-1)}\left(\beta p^{k-1}\right)\right)\right)-\lambda x\right\}
\end{aligned}
$$

and where $W(\lambda, t, p)$ is defined in equation (10).

Proof. The proof of Theorem 3.1 follows directly from Lemma 3.1 and Chebyshev's inequality.

Choosing a specific sequence $q_{k}$ in Lemma 3.1, we obtain the following result.

Lemma 3.2. Let $f=\{f(t), t \in B\}$ be a continuous function such that

$$
|f(u)-f(w)| \leq \delta(\rho(u, w))
$$


where $\delta=\{\delta(s), s>0\}$ is an increasing nonnegative function. Further let

$$
\int_{0}^{\beta} \zeta_{\varphi}\left(H_{B_{t}}\left(\sigma^{(-1)}(u)\right)\right) d u<\infty .
$$

Then

$$
\begin{aligned}
\operatorname{E} \exp \{\lambda Q(t)\} \leq & W_{1}(\lambda, t, p) \\
& \times \exp \left\{\varphi\left(\frac{\lambda \beta}{1-p}\right) p+\frac{2 \lambda}{p(1-p)} \int_{0}^{\beta p^{2}} \zeta_{\varphi}\left(H_{B_{t}}\left(\sigma^{(-1)}(u)\right)\right) d u\right\} \\
& \times \exp \left\{\lambda \sum_{k=1}^{\infty} \delta\left(\sigma^{(-1)}\left(\beta p^{k-1}\right)\right)\right\}
\end{aligned}
$$

for all $\lambda>0$ and $p \in(0,1)$, where

$$
W_{1}(\lambda, t, p)=\inf _{v \geq \frac{1}{1-p}} \min \left\{X_{v, \lambda, t, p}, Y_{v, \lambda, t, p}\right\}
$$

and

$$
\begin{aligned}
X_{v, \lambda, t, p}=\left(\int_{1}^{N_{B_{t}}\left(\sigma^{(-1)}(\beta p)\right)+1} \exp \left\{\varphi\left(v \lambda \sigma\left(2 x \sigma^{(-1)}(\beta p)\right)\right)+v \lambda \delta\left(2 x \sigma^{(-1)}(\beta p)\right)\right\} d x\right)^{\frac{1}{v}}, \\
Y_{v, \lambda, t, p}=\left(N_{B_{t}}\left(\sigma^{(-1)}(\beta p)\right)\right)^{\frac{1}{v}} \\
\quad \times \inf _{w>1} \exp \left\{\frac{\psi(w v \lambda \gamma(t))}{w v}\right. \\
\left.\quad \times \max _{u \in B_{t}}\left\{\frac{(w-1) \psi\left(\frac{w v}{w-1} \lambda \gamma(u)\right)}{w v}+\lambda f(u)\right\}-\lambda f(t)\right\} .
\end{aligned}
$$

Proof. Inequality (9) implies that

$$
\begin{aligned}
E \exp \{\lambda Q(t)\} \leq & W(\lambda, t, p) \\
& \times \exp \left\{\lambda \sum_{k=2}^{\infty} \delta\left(\sigma^{(-1)}\left(\beta p^{k-1}\right)\right)\right\} \\
& \times \exp \left\{\sum_{k=2}^{\infty} \frac{H_{B_{t}}\left(\varepsilon_{k}\right)+\varphi\left(\lambda q_{k} \beta p^{k-1}\right)}{q_{k}}\right\}
\end{aligned}
$$

for all $q_{k}, k \geq 1$, such that

$$
q_{k}>1, \quad k \geq 1, \quad \sum_{k=1}^{\infty} \frac{1}{q_{k}} \leq 1,
$$

and for all $\lambda>0$, where $\varepsilon_{k}=\sigma^{(-1)}\left(\beta p^{k}\right)$.

Let $v$ be a number such that $v \geq(1-p)^{-1}$ and put $q_{1}=v$. Note that $q_{1}>1$. Further let, for $k=2,3, \ldots$,

$$
q_{k}=\frac{1}{\lambda \beta p^{k-1}} \varphi^{(-1)}\left(\varphi\left(\frac{\lambda \beta}{1-p}\right)+H_{B_{t}}\left(\varepsilon_{k}\right)\right) .
$$

Then

$$
q_{k}>\frac{1}{p^{k-1}(1-p)}>1, \quad k=2,3, \ldots
$$


Since

$$
\frac{1}{q_{k}} \leq \frac{\lambda \beta p^{k-1}}{\varphi^{(-1)}\left(\varphi\left(\frac{\lambda \beta}{1-p}\right)\right)}=p^{k-1}(1-p), \quad k \geq 2,
$$

we have

$$
\sum_{k=1}^{\infty} \frac{1}{q_{k}} \leq \sum_{k=1}^{\infty} p^{k-1}(1-p)=1 \text {. }
$$

Thus conditions (24) hold.

Now we consider

$$
\tilde{Z}=\sum_{k=2}^{\infty} \frac{H_{B_{t}}\left(\varepsilon_{k}\right)+\varphi\left(\lambda q_{k} \beta p^{k-1}\right)}{q_{k}} .
$$

For the sequence $q_{k}$ defined by (25), we get

$$
\begin{aligned}
\tilde{Z} & =\sum_{k=2}^{\infty} \frac{H_{B_{t}}\left(\varepsilon_{k}\right)}{q_{k}}+\sum_{k=2}^{\infty} \frac{1}{q_{k}} \varphi\left(\lambda \beta p^{k-1} \frac{\varphi^{(-1)}\left(\varphi\left(\frac{\lambda \beta}{1-p}\right)+H_{B_{t}}\left(\varepsilon_{k}\right)\right)}{\lambda \beta p^{k-1}}\right) \\
& =\sum_{k=2}^{\infty} \frac{H_{B_{t}}\left(\varepsilon_{k}\right)}{q_{k}}+\sum_{k=2}^{\infty} \frac{H_{B_{t}}\left(\varepsilon_{k}\right)}{q_{k}}+\varphi\left(\frac{\lambda \beta}{1-p}\right) \sum_{k=2}^{\infty} \frac{1}{q_{k}} \\
& \leq 2 \sum_{k=2}^{\infty} H_{B_{t}}\left(\varepsilon_{k}\right) \frac{\lambda \beta p^{k-1}}{\varphi^{(-1)}\left(H_{B_{t}}\left(\varepsilon_{k}\right)\right)}+\varphi\left(\frac{\lambda \beta}{1-p}\right) \sum_{k=2}^{\infty} p^{k-1}(1-p) \\
& =\varphi\left(\frac{\lambda \beta}{1-p}\right) p+2 \lambda \sum_{k=2}^{\infty} \zeta_{\varphi}\left(H_{B_{t}}\left(\sigma^{(-1)}\left(\beta p^{k}\right)\right)\right) \beta p^{k-1} .
\end{aligned}
$$

The function $\varphi(x) / x$ increases for $x>0$. Thus the function $\zeta_{\varphi}(x)=x / \varphi^{(-1)}(x)$ increases for $x>0$, too. Then

$$
\int_{\beta p^{k+1}}^{\beta p^{k}} \zeta_{\varphi}\left(H_{B_{t}}\left(\sigma^{(-1)}(u)\right)\right) d u \geq \zeta_{\varphi}\left(H_{B_{t}}\left(\sigma^{(-1)}\left(\beta p^{k}\right)\right)\right) \beta p^{k}(1-p) .
$$

Relations (26) and (27) imply that

$$
\tilde{Z} \leq \varphi\left(\frac{\lambda \beta}{1-p}\right) p+\frac{2 \lambda}{p(1-p)} \int_{0}^{\beta p^{2}} \zeta_{\varphi}\left(H_{B_{t}}\left(\sigma^{(-1)}(u)\right)\right) d u .
$$

Therefore inequality (21) follows from (9) and (28).

Choosing the sequence $q_{k}$ defined in Lemma 3.2, we obtain the following result.

Theorem 3.2. Let a stochastic process $X(t)=\{X(t), t \in B\}$ belong to the class $V(\varphi, \psi)$ and let $\varphi \prec \psi$. Assume that condition (7) holds. Let $f=\{f(t), t \in B\}$ be a continuous function such that

$$
|f(u)-f(w)| \leq \delta(\rho(u, w)),
$$

where $\delta=\{\delta(s), s>0\}$ is an increasing nonnegative function. Further let

$$
r=\{r(u): u \geq 1\}
$$

be a continuous function such that $r(1)=0, r(u)>0$ for $u>1$, and the function $s(t)=r(\exp \{t\}), t \geq 0$, is convex. If

$$
\int_{0}^{\beta} \frac{r\left(N_{B_{t}}\left(\sigma^{(-1)}(u)\right)\right)}{\varphi^{(-1)}\left(H_{B_{t}}\left(\sigma^{(-1)}(u)\right)\right)} d u<\infty
$$


for the process $Q(t)$ defined by (8), then

$$
\mathrm{P}\{Q(t)>x\} \leq Z_{r}(p, t, x)
$$

for all $p \in(0,1)$ and $x>0$, where

$$
\begin{aligned}
Z_{r}(p, t, x)=\inf _{\lambda>0} W_{1}(\lambda, t, p) \exp \{ & \left.p \varphi\left(\frac{\lambda \beta}{1-p}\right)+\lambda\left(\sum_{k=2}^{\infty} \delta\left(\sigma^{(-1)}\left(\beta p^{k-1}\right)\right)-x\right)\right\} \\
& \times\left(r^{(-1)}\left(\frac{\lambda}{p(1-p)} \int_{0}^{\beta p^{2}} \frac{r\left(N_{B_{t}}\left(\sigma^{(-1)}(u)\right)\right)}{\varphi^{(-1)}\left(H_{B_{t}}\left(\sigma^{(-1)}(u)\right)\right)} d u\right)\right)^{2}
\end{aligned}
$$

and where $W_{1}(\lambda, t, p)$ is defined by (22).

Theorem 3.2 follows from Lemmas 3.1 and 3.2 The proof is similar to that of Theorem 3.2 of the paper 12 or to that of Theorem 3.4 in 7 .

Using the sequence $q_{k}=1 /\left((1-p) p^{k-1}\right)$ in inequalities (9) we get the following result. An advantage of this result is that its conditions are easier to check than those of Theorem 3.2 .

Theorem 3.3. Let $f=\{f(t), t \in B\}$ be a continuous function such that

$$
|f(u)-f(w)| \leq \delta(\rho(u, w)),
$$

where $\delta=\{\delta(s), s>0\}$ is a nonnegative increasing function and let $r_{1}=\left\{r_{1}(u), u \geq 1\right\}$ be a continuous function such that $r_{1}(u)>0$ for $u>1$ and $s(t)=r_{1}(\exp \{t\}), t \geq 0$, is a convex function. If

$$
\int_{0}^{\beta} r_{1}\left(N_{B_{t}}\left(\sigma^{(-1)}(u)\right)\right) d u<\infty
$$

for the process $Q(t)$ defined by (8), then

$$
\mathrm{P}\{Q(t)>x\} \leq Z_{r_{1}}(p, t, x)
$$

for all $p \in(0,1)$ and $x>0$, where

$$
\begin{aligned}
Z_{r_{1}}(p, t, x)= & \inf _{\lambda>0} W_{2}(\lambda, p, t) \exp \left\{p \varphi\left(\frac{\lambda \beta}{1-p}\right)+\lambda\left(\sum_{k=2}^{\infty} \delta\left(\sigma^{(-1)}\left(\beta p^{k-1}\right)\right)-x\right)\right\} \\
& \times r_{1}^{(-1)}\left(\frac{1}{\beta p} \int_{0}^{\beta p} r_{1}\left(N_{B_{t}}\left(\sigma^{(-1)}(u)\right)\right) d u\right)
\end{aligned}
$$

and

$$
\begin{gathered}
W_{2}(\lambda, t, p)=\min \left\{X_{\lambda, t, p} ; Y_{\lambda, t, p}\right\} \\
X_{\lambda, t, p}=\left(\int_{1}^{N_{B_{t}}\left(\sigma^{(-1)}(\beta p)\right)+1} \exp \left\{\varphi\left(\frac{\lambda \sigma\left(2 x \sigma^{(-1)}(\beta p)\right)}{1-p}\right)+\frac{\lambda \delta\left(2 x \sigma^{(-1)}(\beta p)\right)}{1-p}\right\} d x\right)^{1-p}, \\
Y_{\lambda, t, p}=\left(N_{B_{t}}\left(\sigma^{(-1)}(\beta p)\right)\right)^{1-p} \inf _{v>1} \exp \left\{\frac{(1-p) \psi\left(\frac{v \lambda \gamma(t)}{1-p}\right)}{v}\right. \\
\left.-\lambda f(t)+\max _{u \in B_{t}}\left(\frac{(v-1)(1-p) \psi\left(\frac{v \lambda \gamma(t)}{(v-1)(1-p)}\right)}{v}+\lambda f(u)\right)\right\} .
\end{gathered}
$$

Theorem 3.2 follows from Lemma 3.1. The proof is similar to that of Theorem 3.1 of [12] or that of Theorem 3.5 of [7]. 


\section{An estimate for the distribution of a Stationary $\varphi$-SUb-Gaussian STORAGE PROCESS}

Consider a stationary $\varphi$-sub-Gaussian stochastic process $X=\{X(t), t \in B\}$ defined on a compact set $B \subset T$, where $T$ is a set of parameters. Recall (see [13]) that a $\varphi$-sub-Gaussian process $X$ is called stationary if

1) its norm $\tau_{\varphi}(X(t))=c_{\varphi}=\gamma$ is constant for all $t, s \in B$, and if

2) $\tau_{\varphi}(X(t)-X(s))=\sigma_{\varphi}(t-s)$.

Assume that $X$ satisfies conditions (7). Let a continuous function $f=\{f(t), t \in B\}$ be such that $|f(u)-f(w)| \leq \delta(\rho(u, w))$, where $\delta=\{\delta(s), s>0\}$ is an increasing nonnegative function.

Example 4.1. We apply Lemma 3.2 to $X$. Then we estimate both terms on the right hand side of (22) defining the minimum of the function $W_{1}(\lambda, t, p)$.

First we consider the case of

$$
v=\frac{w-1}{\lambda \gamma w} \varphi^{(-1)}\left(\varphi\left(\frac{\lambda \gamma w}{(1-p)(w-1)}\right)+H_{B_{t}}\left(\sigma^{(-1)}(\beta p)\right)\right) .
$$

Then

$$
v \geq \frac{w-1}{\lambda \gamma w} \varphi^{(-1)}\left(\varphi\left(\frac{\lambda \gamma w}{(1-p)(w-1)}\right)\right)=\frac{1}{1-p}
$$

In this case,

$$
\begin{aligned}
& W_{1}(\lambda, t, p) \\
& \leq\left(N_{B_{t}}\left(\sigma^{(-1)}(\beta p)\right)\right)^{\frac{1}{v}} \\
& \times \inf _{w>1} \exp \left\{\frac{\varphi\left(w \frac{w-1}{\lambda \gamma w} \varphi^{(-1)}\left(\varphi\left(\frac{\lambda \gamma w}{(1-p)(w-1)}\right)+H_{B_{t}}\left(\sigma^{(-1)}(\beta p)\right)\right) \lambda \gamma\right)}{w v}\right. \\
& \left.+\frac{(w-1) \varphi\left(\frac{w}{w-1} \frac{w-1}{\lambda \gamma w} \varphi^{(-1)}\left(\varphi\left(\frac{\lambda \gamma w}{(1-p)(w-1)}\right)+H_{B_{t}}\left(\sigma^{(-1)}(\beta p)\right)\right) \lambda \gamma\right)}{w v}\right\} \\
& =\left(N_{B_{t}}\left(\sigma^{(-1)}(\beta p)\right)\right)^{\frac{1}{v}} \\
& \times \inf _{w>1} \exp \left\{\frac{\varphi\left((w-1) \varphi^{(-1)}\left(\varphi\left(\frac{\lambda \gamma w}{(1-p)(w-1)}\right)+H_{B_{t}}\left(\sigma^{(-1)}(\beta p)\right)\right)\right)}{w v}\right. \\
& \left.+\frac{(w-1)\left(\varphi\left(\frac{\lambda \gamma w}{(1-p)(w-1)}\right)+H_{B_{t}}\left(\sigma^{(-1)}(\beta p)\right)\right)}{w v}\right\} .
\end{aligned}
$$

Now let

$$
v=\frac{1}{\lambda} \varphi^{(-1)}\left(\varphi\left(\frac{\lambda}{1-p}\right)+H_{B_{t}}\left(\sigma^{(-1)}(\beta p)\right)\right) .
$$

Then

$$
v \geq \frac{1}{\lambda} \varphi^{(-1)}\left(\varphi\left(\frac{\lambda}{1-p}\right)\right)=\frac{1}{1-p},
$$


whence

$$
\begin{aligned}
W_{1}(\lambda, t, p) \\
\leq\left(\int _ { 1 } ^ { N _ { B _ { t } } ( \sigma ^ { ( - 1 ) } ( \beta p ) ) + 1 } \operatorname { e x p } \left\{\varphi\left(\frac{1}{\lambda} \varphi^{(-1)}\left(\varphi\left(\frac{\lambda}{1-p}\right)+J(t, p)\right) \lambda \sigma(K(x, p))\right)\right.\right. \\
\left.\left.+\frac{1}{\lambda} \varphi^{(-1)}\left(\varphi\left(\frac{\lambda}{1-p}\right)+J(t, p)\right) \lambda \delta(K(x, p))\right\} d x\right)^{\frac{1}{v}} \\
\exp \left\{\left(\varphi\left(\frac{\lambda}{1-p}\right)+J(t, p) \sigma(K(x, p))\right)\right. \\
+\left(\int_{1}^{N_{B_{t}}\left(\sigma^{(-1)}(\beta p)\right)+1}\right. \\
+\varphi^{\left.\left.(-1)\left(\varphi\left(\frac{\lambda}{1-p}\right)+J(t, p)\right) \delta(K(x, p))\right\} d x\right)^{\frac{1}{v}},}
\end{aligned}
$$

where

$$
\begin{gathered}
J(t, p)=H_{B_{t}}\left(\sigma^{(-1)}(\beta p)\right), \\
K(x, p)=2 x \sigma^{(-1)}(\beta p) .
\end{gathered}
$$

Example 4.2. Let $X$ be a stationary sub-Gaussian stochastic process defined in the interval $B=[a, b],-\infty<a<b<\infty$, and let $\rho(u, v)=|u-v|$. Then

$$
\inf _{u \in B_{t}} \sup _{v \in B_{t}} \rho(u, v)=\frac{t-a}{2}
$$

and

$$
\beta \leq \sigma\left(\frac{b-a}{2}\right)
$$

Thus

whence

$$
N_{B_{t}}(u) \leq \frac{t-a}{2 u}+1
$$

if

$$
N_{B_{t}}(u) \leq \frac{t-a}{u}
$$

$$
u \leq \frac{t-a}{2}
$$

As in Example 4.1, we apply Lemma 3.2 to the process $X$. Since $\psi(u)=\varphi(u)=u^{2} / 2$, the expression in (22) simplifies:

$$
\begin{aligned}
& W_{1}(\lambda, t, p)=\inf _{v \geq \frac{1}{1-p}} \min \left\{\left(\int_{1}^{M(t, p)} \exp \left\{\frac{v^{2} \lambda^{2} \sigma^{2}(K(x, p))}{2}+v \lambda \delta(K(x, p))\right\} d x\right)^{\frac{1}{v}}\right. \\
&\left.(M(t, p)-1)^{\frac{1}{v}} \inf _{w>1} \exp \left\{\frac{v \lambda^{2} \gamma^{2} w^{2}}{2(w-1)}\right\}\right\} \\
& \leq \min \left\{\left(\int_{1}^{M(t, p)} \exp \left\{\frac{\lambda^{2} \sigma^{2}(K(x, p))}{2(1-p)^{2}}+\frac{\lambda \delta(K(x, p))}{1-p}\right\} d x\right)^{1-p} ;\right. \\
&\left.(M(t, p)-1)^{2 \lambda^{2} \gamma^{2}}\right\}
\end{aligned}
$$


where

$$
M(t, p)=\frac{t-a}{\sigma^{(-1)}(\beta p)}+1
$$

and where $K(x, p)$ is defined in Example 4.1. Moreover,

$$
\int_{0}^{\beta p^{2}} \zeta_{\varphi}\left(H_{B_{t}}\left(\sigma^{(-1)}(u)\right)\right) d u=\int_{0}^{\beta p^{2}}\left(2 \ln \left(\frac{t-a}{\sigma^{(-1)}(u)}\right)\right)^{\frac{1}{2}} d u .
$$

Therefore we proved the following result.

Theorem 4.1. Let $X(t)$ be a stationary sub-Gaussian stochastic process defined on a finite interval and let condition (17) hold. Further let $f=\{f(t), t \in B\}$ be a continuous function such that $|f(u)-f(w)| \leq \delta(\rho(u, w))$, where $\delta=\{\delta(s), s>0\}$ is an increasing nonnegative function. If

$$
\int_{0}^{\beta}\left(\ln \left(\frac{t-a}{\sigma^{(-1)}(u)}\right)\right)^{\frac{1}{2}} d u<\infty
$$

for all $p \in(0,1)$ and $x>0$, then

$$
\begin{aligned}
\mathrm{P}\{Q(t)>x\} \leq & \inf _{\lambda>0} W_{3}(\lambda, t, p) \\
& \times \exp \left\{\frac{\lambda^{2} \beta^{2} p}{2(1-p)^{2}}+\frac{\lambda \sqrt{2}}{p(1-p)} \int_{0}^{\beta p^{2}}\left(\ln \left(\frac{t-a}{\sigma^{(-1)}(u)}\right)\right)^{\frac{1}{2}} d u\right\} \\
& \times \exp \left\{\lambda \sum_{k=1}^{\infty} \delta\left(\sigma^{(-1)}\left(\beta p^{k-1}\right)\right)-\lambda x\right\},
\end{aligned}
$$

where $W_{3}(\lambda, t, p)=\min \left\{X_{\lambda, t, p} ; Y_{\lambda, t, p}\right\}$ and where

$$
\begin{gathered}
X_{\lambda, t, p}=\left(\frac{t-a}{\sigma^{(-1)}(\beta p)}\right)^{2 \lambda^{2} \gamma^{2}} \\
Y_{\lambda, t, p}=\left(\int_{1}^{\frac{t-a}{\sigma^{(-1)(\beta p)}}+1} \exp \left\{\frac{\lambda^{2} \sigma^{2}\left(2 x \sigma^{(-1)}(\beta p)\right)}{2(1-p)^{2}}+\frac{\lambda \delta\left(2 x \sigma^{(-1)}(\beta p)\right)}{1-p}\right\} d x\right)^{1-p} .
\end{gathered}
$$

Example 4.3. Let a stochastic process $X$ in Theorem 4.1 be such that $\sigma(u)=u^{1 / 2}$ and let $f(u)=\delta(u)=c u$, where $c>0$ is a constant. Then

$$
\begin{aligned}
W_{3}(\lambda, t, p)= & \min \left\{D(t, p) ;\left(\int_{1}^{\frac{t-a}{\beta^{2} p^{2}}+1} \exp \left\{x\left(\frac{\lambda^{2} \beta p}{(1-p)^{2}}+\frac{2 \lambda \beta^{2} p^{2}}{1-p}\right)\right\} d x\right)^{1-p}\right\} \\
= & \min \left\{D(t, p) ;\left(\frac{(1-p)^{2}}{\lambda \beta p(\lambda+2 \beta p(1-p))}\right)^{1-p} \exp \left\{\lambda \beta p\left(\frac{\lambda}{1-p}+2 \beta p\right)\right\}\right. \\
& \left.\times\left(\exp \left\{\frac{t-a}{\beta p}\left(\frac{\lambda^{2}}{1-p}+2 \lambda\right)\right\}-1\right)\right\},
\end{aligned}
$$

where

$$
D(t, p)=\left(\frac{t-a}{\beta^{2} p^{2}}\right)^{2 \lambda^{2} \gamma^{2}}
$$

Changing the variables

$$
s=\left(\ln \frac{t-a}{u^{2}}\right)^{\frac{1}{2}}
$$


we obtain

$$
\begin{aligned}
& \int_{0}^{\beta p^{2}}\left(\ln \left(\frac{t-a}{\sigma^{(-1)}(u)}\right)\right)^{\frac{1}{2}} d u \\
& =\int_{0}^{\beta p^{2}}\left(\ln \left(\frac{t-a}{u^{2}}\right)\right)^{\frac{1}{2}} d u \\
& =(t-a)^{\frac{1}{2}} \int_{\left(\ln \frac{t-a}{\beta p^{2}}\right)^{\frac{1}{2}}}^{\infty} s^{2} \exp \left\{-\frac{s^{2}}{2}\right\} d s \\
& =(t-a)^{\frac{1}{2}}\left(-\left.s \exp \left\{-\frac{s^{2}}{2}\right\}\right|_{\left(\ln \frac{t-a}{\beta p^{2}}\right)^{\frac{1}{2}}} ^{\infty}+\int_{\left(\ln \frac{t-a}{\beta p^{2}}\right)^{\frac{1}{2}}}^{\infty} \exp \left\{-\frac{s^{2}}{2}\right\} d s\right) \\
& =(t-a)^{\frac{1}{2}}\left(\left(\ln \frac{t-a}{\beta p^{2}}\right)^{\frac{1}{2}} \exp \left\{-\frac{1}{2} \ln \frac{t-a}{\beta p^{2}}\right\}+\frac{\sqrt{\pi}}{2}-\int_{0}^{\left(\ln \frac{t-a}{\beta p^{2}}\right)^{\frac{1}{2}}} \exp \left\{-\frac{s^{2}}{2}\right\} d s\right) \\
& \leq(t-a)^{\frac{1}{2}} \frac{\sqrt{\pi}}{2} \text {. }
\end{aligned}
$$

Finally

$$
\sum_{k=1}^{\infty} \delta\left(\sigma^{(-1)}\left(\beta p^{k-1}\right)\right)=\sum_{k=1}^{\infty} c \beta^{2} p^{2(k-1)}=\frac{c \beta^{2}}{1-p^{2}}
$$

Therefore bound (34) becomes of the following form in the case of a stationary subGaussian process:

$$
\begin{gathered}
\mathrm{P}\{Q(t)>x\} \leq \inf _{\substack{\lambda>0, p \in(0,1)}} \min \left\{X_{\lambda, p, t} ; Y_{\lambda, p, t}\right\} \\
X_{\lambda, p, t}=\left(\frac{t-a}{\beta^{2} p^{2}}\right)^{2 \lambda^{2} \gamma^{2}} ; \\
Y_{\lambda, p, t}=\left(\frac{(1-p)^{2}}{\lambda \beta p(\lambda+2 \beta p(1-p))}\right)^{1-p} \exp \left\{\lambda \beta p\left(\frac{\lambda}{1-p}+2 \beta p\right)\right\} \\
\times\left(\exp \left\{\frac{t-a}{\beta p}\left(\frac{\lambda^{2}}{1-p}+2 \lambda\right)\right\}-1\right) \\
\times \exp \left\{\frac{\lambda^{2} \beta^{2} p}{2(1-p)^{2}}+\frac{\lambda \sqrt{\pi}(t-a)^{\frac{1}{2}}}{\sqrt{2} p(1-p)}+\frac{\lambda c \beta^{2}}{1-p^{2}}-\lambda x\right\} .
\end{gathered}
$$

\section{CONCLUding REMARKS}

We studied the properties of stochastic processes $X$ belonging to the class $V(\varphi, \psi)$ and obtained some bounds for the distribution of the storage process

$$
Q(t)=\sup _{s \leq t}(X(t)-X(s)-(f(t)-f(s)))
$$

generated by $X$, where a continuous increasing function $f(t)$ is the service output rate. The results of the paper can be applied to a wide class of stochastic processes, in particular to Gaussian processes. As an example, the case of stationary sub-Gaussian processes is considered in the paper. 


\section{ACKNOWLEDGMENT}

The authors are grateful to Professor Yu. V. Kozachenko for constant help and valuable comments.

\section{BIBLIOGRAPHY}

1. R. Addie, P. Mannersalo, and I. Norros, Most probable paths and performance formulae for buffers with Gaussian input traffic, Eur. Trans. Telecommun. 13 (3) (2002), 183-196.

2. P. Boulongne, D. Pierre-Loti-Viaud, and V. Piterbarg, On average losses in the ruin problem with fractional Brownian motion as input, Extremes 12 (2009), 77-91. MR2480724 (2010f:60115)

3. V. V. Buldygin and Yu. V. Kozachenko, Metric Characterization of Random Variables and Random Processes, TViMS, Kiev, 1998; English transl., American Mathematical Society, Providence, RI, 2000. MR1743716 (2001g:60089)

4. N. G. Duffield and N. O'Connell, Large deviations and overflow probabilities for the general single-server queue, with applications, Math. Proc. Cambridge Phil. Soc. 118 (1995), 363-374. MR:1341797 (96f:60039)

5. Yu. V. Kozachenko and E. I. Ostrovskii, Banach spaces random variables of sub-Gaussian type, Teor. Veroyatnost. i Mat. Statist. 32 (1985), 42-53; English transl. in Theory Probab. Math. Statist. 32 (1986), 45-56. MR882158(88e:60009)

6. Yu. V. Kozachenko and Yu. A. Kovalchuk, Boundary value problems with random initial conditions, and functional series from $\operatorname{Sub}_{\varphi}(\Omega)$, I, Ukrain. Mat. Zh. 50 (1998), no. 4, 504-515; English transl. in Ukrainian Math. J. 50 (1999), no. 4, 572-585. MR1698149 (2000f:60029)

7. Yu. Kozachenko, O. Vasylyk, and R. Yamnenko, Upper estimate of overrunning by $\operatorname{Sub}_{\varphi}(\Omega)$ random process the level specified by continuous function, Random Oper. and Stoch. Equ. 13 (2005), no. 2, 111-128. MR2152102 (2006b:60207)

8. L. Massoulie and A. Simonian, Large buffer asymptotics for the queue with fractional Brownian input, J. Appl. Probab. 36 (1999), 894-906. MR.1737061 (2000i:60108)

9. I. Norros, A storage model with self-similar input, Queueing Syst. 16 (1994), 387-396. MR 1278465 (95a:60142)

10. I. Norros, On the use of fractional Brownian motions in the theory of connectionless networks, IEEE Journal on selected areas in communications 13 (1995), no. 6, 953-962.

11. R. Yamnenko, Ruin probability for generalized $\varphi$-sub-Gaussian fractional Brownian motion, Theory Stoch. Process. 12 (28) (2006), no. 1-2, 261-275. MR2316577 (2008g:60109)

12. R. Yamnenko and O. Vasylyk, Random process from the class $V(\varphi, \psi)$ : exceeding a curve, Theory Stoch. Process. 13 (29) (2007), no. 4, 219-232. MR2482262 (2010a:60125)

13. Yu. V. Kozachenko, O. I. Vasylyk, and R. E. Yamnenko, $\varphi$-sub-Gaussian Stochastic Processes, Kyiv University, Kyiv, 2008. (Ukrainian)

Department of Probability Theory, Statistics, and Actuarial Mathematics, Faculty for Mechanics and Mathematics, National Taras Shevchenko University, Academician Glushkov Avenue 2, Kiev 03127, Ukraine

E-mail address: yamnenko@univ.kiev.ua

Department of Probability Theory, Statistics, and Actuarial Mathematics, Faculty for Mechanics and Mathematics, National Taras Shevchenko University, Academician Glushkov Avenue 2, Kiev 03127, Ukraine

E-mail address: shes21@ukr.net 